\title{
Initializing a Quantum Register from Mott Insulator States in Optical Lattices
}

\author{
Chuanwei Zhang, V.W. Scarola, and S. Das Sarma ${ }^{1}$ \\ ${ }^{I}$ Condensed Matter Theory Center, Department of Physics, \\ University of Maryland, College Park, Maryland, 20742 USA
}

\begin{abstract}
We propose and quantitatively develop two schemes to quickly and accurately generate a stable initial configuration of neutral atoms in optical microtraps by extraction from the Mott insulator state in optical lattices. We show that thousands of atoms may be extracted and stored in the ground states of optical microtrap arrays with one atom per trap in one operational process demonstrating massive scalability. The failure probability during extraction in the first scheme can be made sufficiently small $\left(\sim 10^{-4}\right)$ to initialize a large scale quantum register with high fidelity. A complementary faster scheme with more extracted atoms but lower fidelity is also developed.
\end{abstract}

PACS numbers: 03.67.Lx

Introduction: Recently quantum computation using neutral atoms has attracted much attention because neutral atoms are well isolated from the environment and may offer a route to scalable quantum computation. Different neutral atom quantum computing proposals [1, 2, 3] differ in trapping techniques. We focus on arrays of microscopic traps (microtraps) that can be implemented using independent focused laser beams. The typical size of a microtrap in current experiments is about $2 \mu \mathrm{m}$ because of the diffraction limit of a laser beam as well as experimentally technical issues. Unlike an optical lattice, all microtraps can be moved independently in position space, therefore they have advantages in single atom addressability and controlled interactions between pairs of atoms. In recent years, many schemes have been proposed for universal quantum computation in microtrap systems [3].

These schemes are based on the assumption that thousands of neutral atoms can be prepared in the ground states of optical microtrap arrays with one atom per trap, i.e. they assume the ability to initialize a neutral atom quantum register. Although impressive experimental progress has been made in trapping single atoms [4], such an assumption has not been fulfilled because the trapping processes are random and not deterministic. Furthermore, the trapped atoms are not in the trap ground states. These difficulties have prevented neutral atom quantum computation architectures from accomplishing the kind of impressive experimental progress recently achieved in ion trap quantum computation [5]. To overcome these difficulties one proposal seeks to extract single atoms from a Bose-Einstein condensate (BEC) by moving an optical dipole trap out of the condensate [6].

In this paper, we propose two concrete schemes to quickly extract thousands of neutral atoms from the Mott insulator (MI) state in optical lattices [7] and prepare them in the ground states of optical microtrap arrays with precisely one atom per trap. In the MI regime, atom number fluctuations at each site are suppressed and a MI state may be obtained with proper experimental parameters, as demonstrated in recent experiments [2, 7, 8]. Indeed, a perfect MI state with one atom per lattice site may be implemented through purification processes [9]. In MI states, atoms are isolated from each other and occupy the ground states of each site making it difficult to realize a low density MI in long lattice spacing $(d)$ laser geometries [10] where the MI energy scales $\left(\sim d^{-2}\right)$ fall below charac- teristic temperatures. On the other hand, the size of a microtrap $(\sim 2 \mu \mathrm{m})$ is much larger than the typical lattice spacing $(\sim 0.4 \mu \mathrm{m})$, which make it difficult to extract atoms to microtraps directly from a MI state. Therefore the main challenge, and also the goal of this paper, is to transfer single atoms from optical lattices with a short lattice period (SPOL) to spatially separate optical microtraps.

We show in the first scheme that such transfer process may be accomplished using hyperfine state dependent optical lattices with a long lattice period (LPOL), microwave radiation, and resonant "removing" lasers. A LPOL is created by intersecting two laser beams at a certain angle, as demonstrated in a recent experiment [10]. The LPOL induces position-dependent energy shifts of the hyperfine states of atoms, which, when combined with microwave radiation and resonant "removing" lasers, expel many atoms out of the optical lattice and form a patterned loaded optical lattice with one atom per $n$ lattice sites ( $n \geq 3$ is an integer). The remaining atoms in the lattices are well separated, and can be adiabatically transferred to the ground states of optical microtrap arrays with one atom per trap. With our scheme, thousands of atoms may be extracted and the failure probability in extraction can be kept below $\sim 10^{-4}$. In the second scheme, the transfer process is accomplished using state-dependent focused lasers without expelling atoms out of the optical lattice, therefore more atoms can be extracted but the failure probability is much higher. These complementary schemes, one with very high fidelity (but relatively lower speed) and the other with very fast speed (but relatively lower fidelity), take advantage of the robust localization inherent in the MI to isolate atoms. The whole operational process is simple and within currently accessible experimental technology.

The first extraction scheme includes four operational steps and their time sequences are schematically plotted in Fig. 1a. In the following, we explain each step and study various errors that could lead to failures in extraction. For simplicity, we focus on a one dimensional geometry but we emphasize that our technique can be straightforwardly applied to two dimensional arrays.

Step (I) Initial Mott state: Consider a pure ${ }^{87} \mathrm{Rb}$ BEC prepared in the hyperfine ground state $|0\rangle \equiv\left|F=1, m_{F}=-1\right\rangle$ and confined in a quasi-one dimensional ( $x$ direction) harmonic magnetic trap. Along the transverse direction, the 


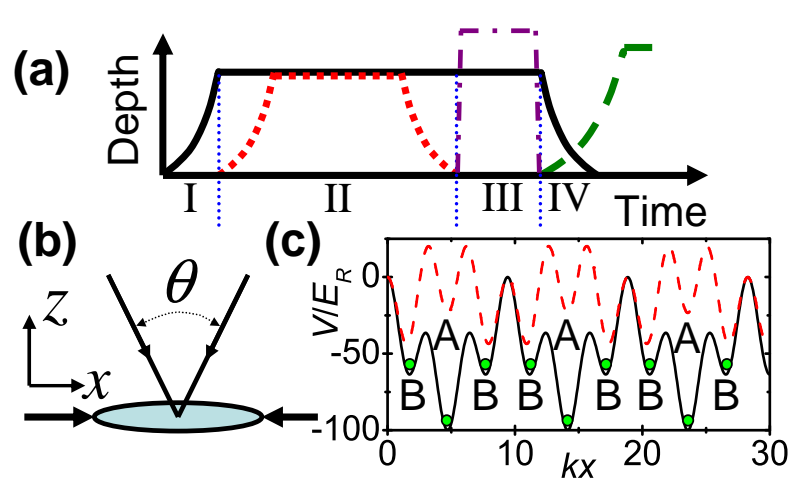

Figure 1: (color online). Schematic plot of the single atom extraction. (a) Time sequence for laser depths at four extraction steps. Solid line (SPOL), dotted line (LPOL), dashed dotted line (removing laser), dashed line (microtrap laser). (b) Geometry of additional lasers defining the LPOL. (c) Site specific energies in the optical lattice. Solid and dashed lines correpsond to the optical potentials for atoms at states $|0\rangle$ and $|1\rangle$ respectively. $A$ labels target atoms for extraction and $B$ labels other atoms.

atomic dynamics are frozen out by high frequency optical traps [11]. An optical lattice along the $x$ direction with wavelength $\lambda_{s}=850 \mathrm{~nm}$ is ramped up adiabatically $(\sim 200 \mathrm{~ms})$ to a large potential depth of $V_{s}=50 E_{R}$ such that the BEC is converted into a MI state with roughly one atom per lattice site, using properly chosen trapping parameters and number of atoms [7]. Here $E_{R}=h^{2} / 2 m \lambda_{s}^{2}$ denotes the recoil energy. This MI state may have defects [8] and clever purification schemes [9] may yield a nearly perfect MI state with exact one atom per lattice site. Such a perfect MI state is the reservoir for single atom extraction and provides the starting point of our scheme.

Step (II) Selective Depopulation: Here atoms at specific, unwanted sites are transferred to another hyperfine state. Two $\sigma^{+}$-polarized laser beams intersecting at an angle $\theta$ (Fig.1b), which drive the $5 S \rightarrow 5 P$ transition and has a detuning $\Delta_{2}=-2 \pi \times 3608 G H z$ to the $5^{2} P_{3 / 2}$ state (corresponding to a wavelength $\lambda_{l}=787.6 \mathrm{~nm}$ ), are adiabatically ramped up. These two beams form a LPOL along the $x$ direction with the lattice period $\eta_{l}=\lambda_{l} /$ [2 $\sin (\theta / 2)$ ]. Here we require $\eta_{l}$ to be $n$ times that of the short wavelength optical lattice period $\lambda_{s} / 2$, that is, $\theta=2 \arcsin \left[\lambda_{l} / n \lambda_{s}\right]$. The LPOL induces energy shifts

$$
\delta E_{ \pm}(r)=\frac{3 \pi c^{2} I(r)}{2} \sum_{q=1,2} \frac{\Gamma_{q}\left|c_{ \pm q}\right|^{2}}{\omega_{q}^{3} \Delta_{q}}
$$

for two finestructure ground states $| \pm\rangle=$ $\left|5 S: j=1 / 2, m_{j}= \pm 1 / 2\right\rangle$, where $I(r)$ is the intensity of the laser, $\Gamma_{q}(q=1,2)$ is the decay rate for states $5^{2} P_{1 / 2}$ and $5^{2} P_{3 / 2}$. $\omega_{q}\left(\Delta_{q}\right)$ is the frequency (detuning) for the transition from $5 S$ to $5^{2} P_{1 / 2}$ and $5^{2} P_{3 / 2} . c_{+1}=0, c_{+2}=1$, $c_{-1}=-\sqrt{2 / 3}, c_{-2}=\sqrt{1 / 3}$ are transition coefficients. Two hyperfine states $|0\rangle$ and $|1\rangle \equiv|F=2, m=-2\rangle$ can be written as $|0\rangle=1 / 4|-\rangle+3 / 4|+\rangle,|1\rangle=|-\rangle$ using the respective Clebsch-Gordan coefficients. Therefore the energy shifts for states $|0\rangle$ and $|1\rangle$ are $\delta E_{0}=\delta E_{-} / 4+3 \delta E_{+} / 4$, and $\delta E_{1}=\delta E_{-}$, which give the total shift of the hyperfine splitting between $|0\rangle$ and $|1\rangle, \delta E=\delta E_{1}-\delta E_{0}=\frac{\alpha_{1}}{2 \Delta_{1}}-\frac{\alpha_{2}}{2 \Delta_{2}}$, where $\alpha_{q}=3 \pi c^{2} \Gamma_{q} I / 2 \omega_{q}^{3}$. Following a similar procedure, we find the spontaneous emission rates for atoms at $|0\rangle$ and $|1\rangle$ are

$$
\gamma_{0}=\frac{\alpha_{1} \Gamma_{1}}{6 \Delta_{1}^{2}}+\frac{5 \alpha_{2} \Gamma_{2}}{6 \Delta_{2}^{2}}, \gamma_{1}=\frac{2 \alpha_{1} \Gamma_{1}}{3 \Delta_{1}^{2}}+\frac{\alpha_{2} \Gamma_{2}}{3 \Delta_{2}^{2}} .
$$

Notice that the wavelength $\lambda_{l}=787.6 \mathrm{~nm}$ (i.e. the detuning $\left.\Delta_{2}=-2 \pi \times 3608 G H z\right)$ is optimized to obtain the maximal ratio $\eta=\delta E / \gamma$ between the shift $\delta E$ and the rate $\gamma=\max \left\{\gamma_{0}, \gamma_{1}\right\}$.

The energy shifts induced by the LPOL are spatially dependent for atoms confined in the SPOL. In Fig. 1c, we plot the combined lattice potentials for atoms at $|0\rangle$ and $|1\rangle$ with $n=3$. We see that the LPOL induces two different shifts of the hyperfine splitting, depending on the positions of atoms ( $A$ or $B$ ), and the difference $\delta=\delta E(A)-\delta E(B)$ can be adjusted and chosen to be $\delta=52 E_{R}$. Applying the adiabatic condition, we estimate the ramp up time to be $44 \mu s$ that corresponds to a $10^{-4}$ probability for excitation to higher bands.

We then apply a microwave $\pi$ pulse to flip the quantum states of atoms at position $B$ (Fig. 1c) from $|0\rangle$ to $|1\rangle$. The microwave is resonant with the hyperfine splitting of atoms at $B$, but has a detuning $\delta$ for atoms at $A$. A time-dependent microwave pulse with frequency $\Omega(t)=\Omega_{0} \exp \left(-\omega_{0}^{2} t^{2}\right)\left(-t_{f} \leq\right.$ $\left.t \leq t_{f}\right)$ is used to perform the $\pi$ pulse. For a set of microwave parameters $\omega_{0}=\delta / 4=13 E_{R} / \hbar, t_{f}=5 / \omega_{0}$, and $\Omega_{0}=\pi /\left[\int_{-t_{f}}^{t_{f}} \exp \left(-\omega_{0}^{2} t^{2}\right) d t\right] \approx 23 E_{R} / \hbar$, the pulse flips the quantum state of atom $B$ from $|0\rangle$ to $|1\rangle$ in $2 t_{f}=38.6 \mu \mathrm{s}$, while the error to flip atom $A$ to state $|1\rangle$ is found to be $5.9 \times 10^{-6}$ by numerically integrating the Rabi equation [12] that describes the coupling between $|0\rangle$ and $|1\rangle$ through the microwave pulse. The LPOL are adiabatically turned off after the microwave pulse. During the whole process time $\tau$, the probability for spontaneous scattering of one photon from each atom is estimated to be $P=\int_{0}^{\tau} \gamma d t \approx 1 \times 10^{-4}$.

Step (III) Remove Non-target Atoms: In this step, atoms at $B$ are removed from the trap by applying a $\sigma^{-}$-polarized "removing" laser that drives a resonant cycling transition $|1\rangle \rightarrow$ $|2\rangle \equiv\left|5^{2} P_{3 / 2}: F=3, m_{F}=-3\right\rangle$. Scattering photons from the laser push (or heat) non-target atoms $B$ at $|1\rangle$ out of the trap without affecting target atoms $A$ at state $|0\rangle$ because of the large hyperfine splitting $(v \approx 2 \pi \times 6.8 G \mathrm{~Hz})$ between the two states. To push (or heat) atoms out of a trap with depth $U_{0}$, the number of spontaneous emission photons needs to be at least $n_{p}=U_{0} / 2 E_{R}[4]$. For instance, 25 photons are needed for each non-target atom to remove it from an optical lattice with depth $U_{0}=50 E_{R}$. The dynamics of the photon scattering process are described by the optical Bloch equation [12], from which we can numerically calculate the number of scattering photons $n_{p}$ for both target and non-target atoms. We find the number of scattering photons can reach 25 in a short period $\sim 1 \mu \mathrm{s}$ for atoms $B$, but it is only $\sim 10^{-5}$ for atoms $A$. Therefore the impact of the resonant laser on the target atoms $A$ can be neglected. Note that hot non-target atoms tunnel more easily in the optical lattice, which enhances the collision probability between target and non-target atoms at 
different lattice sites. However, because of the high la depths $\left(\sim 50 E_{R}\right)$, the tunneling rate is quite low $(\sim 0.01$ on average for hot non-target atoms, which yields a long neling time $(\sim 100 \mathrm{~ms})$. The short lifetime $(\sim 1 \mu \mathrm{s})$ of non-target atoms makes the collision probability very $\mathrm{s}$ $\left(\sim 1 \mu \mathrm{s} / 100 \mathrm{~ms}=10^{-5}\right)$. The effect of interatomic collis on the target atoms can therefore be neglected.

Step (IV) Transfer to Microtraps: In this last step, the maining target atoms $A$ at the optical lattice sites are a batically distributed to the ground state of optical micrc arrays with one atom per trap. The optical microtraps be focused far red-detuned lasers or high frequency $\mathrm{b}$ detuned optical traps using Hermite-Gaussian TEM T1 $\mathrm{n}$ beams [11]. Each optical microtrap is focused near one get atom and contains only one atom because of the 1 spacing between atoms in the lattice. In the distribution cess, the effective potentials the target atoms experience be approximated as harmonic potentials with the trapping frequency $\varpi(t)=\sqrt{\left(4 V_{f}(t) / w^{2}+2 V_{L}(t) k^{2}\right) / m}$, where $V_{f}(t)$ and $V_{L}(t)$ are the potential depths of the microtraps and the optical lattice respectively, $w$ is the beam waist of the microtrap lasers. $V_{f}(t)$ and $V_{L}(t)$ may be varied simultaneously to adjust $\varpi(t)$ from its initial value $\varpi(0)=\left(2 E_{R} / \hbar\right) \sqrt{V_{L}(0) / E_{R}}$ to the final expected $\varpi\left(t_{0}\right)$. If $\varpi\left(t_{0}\right)=\varpi(0)$, the final microtrap potential depth $V_{f}\left(t_{0}\right)=V_{L}(0) k^{2} w^{2} / 2 \approx 1366 E_{R}$ $(\sim 104 \mu K)$ for initial parameters $V_{s}(0)=50 E_{R}$, and $w=1 \mu m$.

When a deeper or shallower microtrap potential depth is needed, the trapping frequency $\varpi(t)$ should be adjusted so that the adiabatic condition is satisfied

$$
\hbar\left|\frac{d \varpi(t)}{d t}\right|=\xi \frac{\left(\Delta E_{g}\right)^{2}}{\left|\left\langle\phi_{e}\left|\frac{\partial H}{\partial \varpi}\right| \phi_{g}\right\rangle\right|},
$$

where $\left|\phi_{g}\right\rangle$, and $\left|\phi_{e}\right\rangle$ are the ground and the excited state wavefunction, $\Delta E_{g}$ is the energy gap between two states, and $\xi$ is the adiabaticity parameter. Because of the parity of the wavefunctions, the lowest possible excitation is to the second excited state, which gives $\Delta E_{g}=2 \hbar \varpi$, and $\left\langle\phi_{e}\left|\frac{\partial H}{\partial \varpi}\right| \phi_{g}\right\rangle=\hbar / \sqrt{2}$. The adiabatic condition, Eq. (3), yields a time-dependent trapping frequency $\varpi(t)=\varpi(0) /(1 \mp 4 \sqrt{2} \xi \varpi(0) t)$, where $\mp$ correspond to a deeper and shallower final trapping frequency respectively.

Under the adiabatic approximation, the quantum states of the target atoms can be expanded using the time-dependent basis $\varphi(t)=c_{g}(t) \phi_{g}(\varpi(t))+c_{e}(t) \phi_{e}(\varpi(t))$, where $\phi_{g}(\varpi(t))$, and $\phi_{e}(\varpi(t))$ are the adiabatic ground and second excited states of the Hamiltonian $H(\varpi(t))$ with the associated eigenenergies $E_{g}=\hbar \varpi(t) / 2$ and $E_{e}=5 \hbar \varpi(t) / 2$. Inserting this expansion into the the Schrödinger equation for a single target atom $A$ yields a coupled equation for the coefficients $c_{g}(t)$ and $c_{e}(t)$ :

$$
i \hbar \frac{d}{d t}\left(\begin{array}{c}
c_{g} \\
c_{e}
\end{array}\right)=\left(\begin{array}{cc}
E_{g} & \varkappa(t) \\
-\varkappa(t) & E_{e}
\end{array}\right)\left(\begin{array}{c}
c_{g} \\
c_{e}
\end{array}\right),
$$

where $\varkappa(t)=-i \hbar \frac{d \varpi(t)}{d t} \frac{\left\langle\phi_{e}\left|\frac{\partial H}{\partial \omega}\right| \phi_{g}\right\rangle}{\Delta E_{g}}=i \xi \Delta E_{g}$. This equation can be solved analytically to give the occupation probability at the
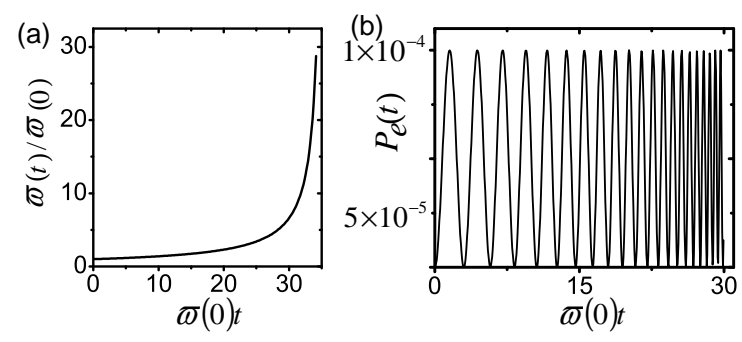

Figure 2: The adiabatic process for the distribution of the target atoms from the optical lattice to arrays of optical dipole microtraps with higher trapping frequencies. Adiabaticity parameter is $\xi=0.005$. (a) Trapping frequency with respect to time. (b) The probability $P_{e}(t)=\left|c_{e}\right|^{2}$ for atoms to be at the second excited state of the effective harmonic trap with respect to time.

second excited states

$$
P_{e}(t)=4 \xi^{2} \sin ^{2}(\ln (1 \mp 4 \sqrt{2} \xi \varpi(0) t) / 4 \sqrt{2} \xi) .
$$

In Fig. 2, we plot the trapping frequency $\varpi(t)$ and excitation probability $P_{e}(t)$ with respect to time for a deeper final trap. We see $P_{e}(t)$ oscillates with time, but has the maximum $4 \xi^{2}$. For adiabaticity parameter $\xi=0.005$, the maximal excitation probability is $10^{-4}$. The total distribution time is $T=(1-\varpi(0) / \varpi(T)) /(4 \sqrt{2} \xi \varpi(0))$. For instance, $T \approx$ $94 \mu s$ is needed to transfer target atoms from the optical lattice to microtraps with trapping frequency $\varpi(T)=4 \varpi(0)$ (corresponding to a $1.66 \mathrm{mK}$ trap potential) with $10^{-4}$ excitation probability. We note that this transfer time is much shorter than the characteristic hopping time $\left(\sim 5 s\right.$ for $\left.V_{s}=50 E_{R}\right)$ of atoms within the depleted optical lattice.

Combining all four operational steps, we find that thousands of atoms can be extracted from the optical lattice to microtraps in less than $300 \mu \mathrm{s}$. For instance, in a one dimensional optical lattice with 300 usable atoms for single atom extraction, we can extract 100 atoms (one per three lattice sites). In a two dimensional trap, the same process can extract $1 / 9$ of total atoms $\left(\sim 10^{4}\right)$ simultaneously, which offers a large scale up in the initialization stage of a quantum computation using microtrap arrays.

The failure probability of extraction does not decrease with an increasing $n$. That is because the microtrap potential is already very weak at $\mathrm{a} \sim 1.3 \mu \mathrm{m}$ displacement (the position of the neighboring atoms in a $n=3$ superlattice) from the trap center for a typical $\sim 2 \mu \mathrm{m}$ microtrap. The potential has a negligible effect on the process of transferring neighboring atoms to other microtraps. A large $n$ lattice does not contribute to the largest error source of the scheme: the heating due to spontaneously scattered photons from atoms in the state-dependent lattice in step (II).

A Speedup Scheme: In the above scheme, the qubit supply time for quantum computation is limited by the period for preparing Bose-Einstein condensates, which is typically on the order of minutes. Notice that in step (III) most atoms (2/3 in $1 \mathrm{D}$ or $8 / 9$ in $2 \mathrm{D}$ lattices) are lost from the lattice by applying the resonant "removing" laser. In the following, we propose a scheme for single atom extraction without remov- 

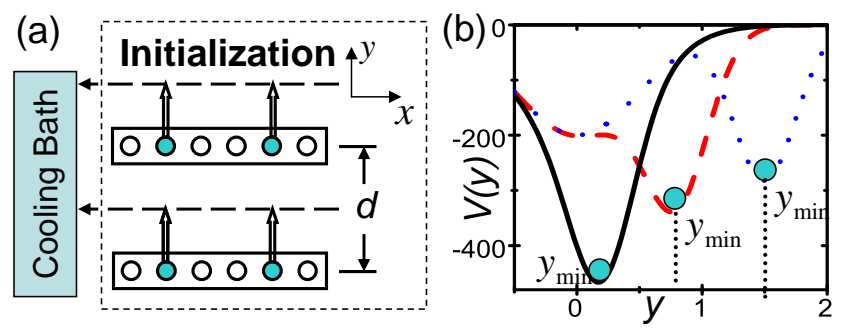

Figure 3: (color online) (a) Schematic plot for the single atom extraction from paralell one dimensional lattices. The distance $d=5 \mu \mathrm{m}$. Filled and opened circles correspond to target and non-target atoms respectively. (b) Optical potentials for target atoms along the $y$ direction. The length and energy units are $\sigma_{c}$ and $\hbar^{2} / 2 m \sigma_{c}^{2}$ respectively. $a(t)=0.2 \sigma_{c}($ solid line $) ; a(t)=0.8 \sigma_{c}($ dashed line $) ; a(t)=1.5 \sigma_{c}$ (dotted line).

ing non-target atoms, which can then be recycled for further extraction processes. In this scheme, $87 \%$ of the atoms can be extracted within several superfluid-insulator transition cycles $(\sim 1 s)$, considerably speeding up the rate for supplying fresh qubits for a neutral atom quantum computer. However, the scheme induces much larger spontaneous emission probability of photons and excitation probability to high motional states for atoms, which significantly degrades the fidelity of the initial qubit, therefore further cooling is needed to obtain qubits with high fidelity.

A schematic for the speed up scheme is plotted in Fig. 3a. Consider a series of parallel well separated $(d=5 \mu \mathrm{m}) 1 \mathrm{D}$ optical lattices (along the $x$ direction) in the $x y$ plane. In the speedup scheme, steps (I)-(II) are still preformed to place all target atoms for extraction into state $|0\rangle$ and non-target atoms to $|1\rangle$. We then ramp up a focused laser with the detuning $\Delta_{0}=-2 \pi \times 780 \mathrm{GHz}$ from the $5 S \rightarrow 6^{2} P_{3 / 2}$ transition so that the focused lasers only induce red-detuned traps for target atoms at state $|0\rangle$, but do not affect non-target atoms at state $|1\rangle[13]$. The focused lasers adiabatically move along $y$ direction to take target atoms out of the optical lattices, without affecting non-target atoms at state $|1\rangle$ (Fig.3a). The atoms inside the focused lasers are then adiabatically transferred to far-detuned dipole traps to suppress spontaneous emission of photons. These dipole traps are moved along the $x$ direction to a cooling bath to improve the fidelity of the initial qubits. At the same time, the Mott insulator states in the optical lattices are melted by adiabatically ramping down the depth of the optical lattice. The trap parameters are adjusted so that another Mott state with one atom per lattice site can be obtained as the lattice depths are adiabatically ramped up. Repeating the above steps, we can extract another $1 / 3$ of the remaining atoms. After 5 such cycles, about $1-(2 / 3)^{5} \approx 87 \%$ atoms can be extracted. Finally, the remaining atoms inside the optical lattices are discarded and a new BEC must be produced to continue the process.

To extract the atoms adiabatically from optical lattices avoiding excitations to higher bands of the focused laser traps, high potential depths are needed, which leads to high spontaneous photon scattering probabilities. Therefore a good strat- egy balances these two sources for the degradation of initial qubit fidelity. Assume that the optical potentials along the $y$ direction for target atoms are

$$
\begin{aligned}
V(y)= & -V_{c} \exp \left(-2 y^{2} / \sigma_{c}^{2}\right) \\
& -V_{f} \exp \left(-2(y-a(t))^{2} / \sigma_{f}^{2}\right),
\end{aligned}
$$

where $V_{c}$ and $V_{f}$ are the potential depths for the $1 \mathrm{D}$ confinement and the focused laser, respectively. Gaussian beam approximations with waists $\sigma_{c}$ and $\sigma_{f}$ have been used for a rough estimate. $a(t)$ is the position of the focused laser center and its rate of change should satisfy the adiabatic condition $\hbar|d a(t) / d t|=\bar{\xi} \Delta E_{g}^{2} /\left|\left\langle\phi_{e}|\partial H / \partial a| \phi_{g}\right\rangle\right|$, where the energy gap $\Delta E_{g}$ and transition matrix element $\left|\left\langle\phi_{e}|\partial H / \partial a| \phi_{g}\right\rangle\right|$ can be evaluated for different potentials $V(a(t))$ by solving the single particle Schrödinger equation. The potential minimum position $y_{\min }$ for the target atom is determined through $\partial V / \partial y=0$. The wavefunction of the target atom is expanded in a harmonic oscillator basis $\Psi_{n}(y)=$ $(\varkappa / \pi)^{1 / 4} \exp \left(-\varkappa\left(y-y_{\min }\right)^{2} / 2\right) H_{n}(\sqrt{\varkappa} y) / \sqrt{2^{n} n !}$ around the potential minimum $y_{\min }$, where the oscillation frequency $\varkappa=$ $\left(\partial^{2} V / \partial y^{2}\right)_{y \min } / m$ and $H_{n}(\sqrt{x} y)$ is the Hermite polynomial. The Hamiltonian for the target atoms evaluated in this basis for different $a(t)$ gives the matrix representation $H_{n m}(a(t))=$ $\left\langle\Psi_{n}|H| \Psi_{m}\right\rangle$ ( $n, m \leq 10$ is enough for our calculation). Diagonalization of the matrix yields the eigenenergies and eigenfunctions, which determine $\Delta E_{g}$ and $\left|\left\langle\phi_{e}|\partial H / \partial a| \phi_{g}\right\rangle\right|$ for different $a(t)$. The total moving time can be estimated using

$$
T=\int_{0}^{a_{f}} \hbar\left|\left\langle\phi_{e}|\partial H / \partial a| \phi_{g}\right\rangle\right| / \bar{\xi} \Delta E_{g}^{2} d a
$$

where $a_{f}$ is the final position of the focused laser.

In Fig.3b, we plot the optical potential $V(y)$ for a set of parameters $\sigma_{c} \approx 0.93 \mu m, \sigma_{f} \approx 0.46 \mu m, V_{c}=200 \hbar^{2} / m \sigma_{c}^{2}$, $V_{f}=280 \hbar^{2} / m \sigma_{c}^{2}$ and three different $a(t)$. Applying the above procedure with these parameters, we estimate the extraction time $\sim 5 \mathrm{~ms}$ and the excitation probability to high bands $\sim$ $7 \times 10^{-3}$, with the spontaneous scattering probability $\sim 10^{-2}$. We see that the fidelity of the atoms is not as high as that for the original scheme. Therefore further cooling is needed to significantly improve the fidelity of initial qubits.

Conclusion: We propose two schemes for extracting thousands of atoms simultaneously from the Mott insulator state in optical lattices to optical microtrap arrays with one atom per trap. The extracted atoms stay at the ground states of the microtraps. In the first scheme, about $11 \%$ of atoms from a BEC can be extracted and the failure probability is $\sim 10^{-4}$ with properly chosen experimental parameters. In the speedup scheme, about $87 \%$ of atoms from a BEC can be extracted, but the failure probability is much higher $\sim 10^{-2}$. We provide a detailed quantitative analysis validating the feasibility of our proposed schemes for neutral atom quantum computation.

We thank S.L. Rolston for valuable discussion. This work is supported by ARO-DTO, ARO-LPS, LPS-NSA. 
[1] P. Zoller, et al., quant-ph/0405025; D. Jaksch, et al., Phys. Rev. Lett. 82, 1975 (1999); L.-M. Duan, et al., ibid 91, 090402 (2003); G.K. Brennen, et al., ibid 82, 1060 (1999).

[2] O. Mandel, et al., Phys. Rev. Lett. 91, 010407 (2000); Nature 425, 937 (2003).

[3] T. Calarco, et al., Phys. Rev. A, 61, 022304 (2000); K. Eckert, et al., ibid, 66, 042317 (2002); J. Mompart, et al., Phys. Rev. Lett., 90, 147901 (2003); D. Schrader, et al., ibid 93, 150501 (2004); M. Schulz, et al., quant-ph/0606018

[4] S. Kurl, et al. , Science 293, 278 (2001); N. Schlosser, et al. , Nature 411, 1024 (2001).

[5] J.I. Cirac, and P. Zoller, Phys. Rev. Lett. 744091 (1995); D. Leibfried, et al., Rev. Mod. Phys. 75, 281 (2003).

[6] R.B. Diener, et al., et al., Phys. Rev. Lett. 89, 070401 (2002); E.B. Kolomeisky, et al., Phys. Rev. A 69, 063401 (2004); B.
Mohring, et al. ibid 71, 053601 (2005).

[7] M. Greiner, et al., Nature 415, 39 (2002); I.B. Spielman, et al., Phys. Rev. Lett. 98, 080404 (2007) .

[8] S. Folling, et al., Phys. Rev. Lett. 97, 060403 (2006).

[9] M. Popp, et al., Fort. Phys. 54686 (2006); P. Rabl, et al., Phys. Rev. Lett. 91, 110403 (2003); M. Popp, et al., New J. Phys. 8, 164 (2006).

[10] S. Peil, et al., Phys. Rev. A, 67, 051603(R) (2003); T. Sleator, et al., Phys. Rev. Lett. 68, 1996 (1992).

[11] T.P. Meyrath, et. al., Optics Express, 13, 2843 (2005); T.P. Meyrath, et. al., Phys. Rev. A, 71, 041604(R) (2005);

[12] H.J. Metcalf and P. van der Straten, Laser cooling and trapping. Springer-Verlag, New York, 1999.

[13] C. Zhang, et. al., Phys. Rev. A 74, 042316 (2006). 